\title{
On Fourier $M_{p}^{q}$ multiplier criteria of Marcinkiewicz type*
}

by

WALTER TREBELS (Darmstadt)

Abstract. Fourier $M_{p}^{q}$ multiplier criteria are given for radial functions on $R^{n}$ in case $1<p \leqslant q<\infty$ and for arbitrary functions if additionally $p<q$; here some results of P. I. Lizorkin [11] and A. Bonami and J. L. Clere [1] are covered. The method of proof consists of a direot reduction to fractional Riesz integrals $(p<q)$ or to singular integrals ( $p=q$ with the aid of Bochner-Riesz kernels; Fourier trans-. forms of the latter ones are generated by appropriate partial integrations of the multi pliers in question. Thus both theories of singular and weakly singular integrals including recent results in weighted. $L^{p}$ spaces can be applied immediately.

1. Introduction. In this paper we want to give further evidence of the central role which is played by (spherical and product) BochnerRiesz kernels in Fourier multiplier theory, in particular by their dilations (see [18]). The Fourier transforms of these kernels result from appropriate partial integration of the multipliers in question; thus the frequently used method of partial integration (summation) is the basis for the following. Here we discuss multipliers of type $(p, q), 1<p \leqslant q<\infty$. If $p<q$, we can quite simply reduce the multiplier problem to a discussion of weakly singular integrals, i.e., to fractional Riesz integrals. If $p=q$ (radial functions), the reduction to singular integrals has to be slightly modified. A further advantage of the present approach is to be seen in the fact that one can also immediately apply recent results of Muckenhoupt-Wheeden [12] and Coifman-Fefferman [4] concerning weakly singular and singular integrals on weighted Lebesgue spaces thus yielding multiplier criteria for weighted $L^{p}$ spaces. We mention that we do not need Littlewood-Paley functions which are used in some deductions of Marcinkiewicz type multiplier criteria.

The following notations will be used: $v, x, y \in R^{n}, s, t, u \in R ; j$ $=\left(j_{1}, \ldots, j_{n}\right)$ denotes a multiindex with non-negative integral $j_{k}$, absolute value $|j|=j_{1}+\ldots+j_{n}, D^{j}=\partial^{|j|} / \partial v_{1}^{j_{1}} \ldots \partial v_{n}^{j_{n}} ; S$ is the set of all infinitely differentiable functions, rapidly decreasing at infinity, $S^{\prime}$ its

*AMS (MOS) Subject Classification: 42A18 
dual. On $L^{1}\left(R^{n}\right)$ define the Fourier transformation by

$$
F[f](v)=f^{\wedge}(v)=(2 \pi)^{-n / 2} \int_{R^{n}} f(x) e^{-i v x} d x .
$$

$m \in S^{\prime}$ is called a multiplier of type $(p, q): m \epsilon M_{p}^{\alpha}$, if

$$
\left\|F^{-1}[m F[f]]\right\|_{q} \leqslant C\|f\|_{p},
$$

$F^{-1}$ denoting the inverse transformation on $S^{\prime}$ and $C$ being independent of $f \epsilon S$.

2. Radial multipliers $(p<q)$. As already mentioned above we will essentially use Bochner-Riesz kernels in the proofs to follow. In case of radial multipliers the spherical version is convenient. For $a \geqslant 0$ it is defined by $\left(J_{u}\right.$ being the Bessel function; see e.g. [16], p. 154, p. 171)

$$
r_{\alpha}(x)=C_{\alpha}|x|^{-\alpha-n / 2} J_{a+n / 2}(|x|), \quad J_{u}(t)=O\left(t^{-1 / 2}\right)
$$

$t \rightarrow \infty, u>-1 / 2$ fixed. Its Fourier transform is given by

$$
r_{a}^{\hat{\alpha}}(v)=\left(\max \left\{1-v^{2}, 0\right\}\right)^{\alpha} .
$$

For sufficiently smooth functions $e(t)$ on $(0, \infty)$ set

$$
N_{i}^{b}(e)=\sup _{t>0}\left|t^{i+b n / 2} e^{(i)}(t)\right|
$$

THEOREM 1. Let $0<b<1, e(t)$ be a function on $(0, \infty)$ such that $N_{0}^{b}(e) \leqslant M$ as well as $N_{k+1}^{b}(e) \leqslant M$, where $k$ is the least integer $>n / 2-n b-1 / 2$ if $0<b \leqslant 1 / 2-1 / 2 n$ and $k=0$ otherwise. Then $e\left(v^{2}\right) \in M_{p}^{q}$, where $1<p<q$ $<\infty$ and $1 / q=1 / p-b$.

Proof. The hypothesis clearly implies

$$
\lim _{t \rightarrow \infty} e(t)=0, \quad \int_{c}^{\infty} t^{k}\left|e^{(k+1)}(t)\right| d t<\infty
$$

for each $c>0$. But this is sufficient (see [17], p. 25) for the representation

$$
e(t)=\frac{ \pm 1}{\Gamma(t+1)} \int_{t}^{\infty}(s-t)^{k} d e^{(k)}(s) \quad(t>0)
$$

With the aid of (2.3) the inverse Fourier transform of $e\left(v^{2}\right)$ in $S^{\prime}$ may be evaluated (here the first interchange of integration order is justi- fied on account of the absolute convergence of the double integral):

$$
\begin{aligned}
\left\langle F^{-1}\left[e\left(v^{2}\right)\right], f\right\rangle & =\left\langle e\left(v^{2}\right), f^{\wedge}(v)\right\rangle \quad(f \in S) \\
& =C \int_{R^{n}} \int_{v^{2}}^{\infty}\left(s-v^{2}\right)^{k} e^{(k+1)}(s) d s f^{\wedge}(v) d v \\
& =C \int_{0}^{\infty} s^{k} e^{(k+1)}(s) \int_{v^{2}<s}\left(1-\frac{v^{2}}{s}\right)^{k} f^{\wedge}(v) d v d s \\
& =\int_{R^{n}} C \int_{0}^{\infty} s^{k} e^{(k+1)}(s) s^{n / 2} r_{k}(\sqrt{s} x) d s f(x) d x .
\end{aligned}
$$

To obtain the last equality we used Parseval's formula and an interchange of integration order (again justified by absolute convergencethe inner integral can be estimated by $C|x|^{(b-1)}$ ). Hence

$$
\begin{aligned}
& \text { (2.4) } \quad F^{-1}\left[e\left(v^{2}\right)\right](x)=C \int_{0}^{\infty} s^{k} e^{(k+1)}(s) s^{n / 2} r_{k}(\sqrt{s} x) d s \\
& \leqslant C|x|^{n(b-1)} N_{k+1}^{b}(e) \int_{0}^{\infty}(\sqrt{s}|x|)^{n(1-b)-1}(\sqrt{s}|x|)^{-k-n / 2} \cdot\left|J_{k+n / 2}(\sqrt{s}|x|)\right||x| \frac{d s}{\sqrt{s}} .
\end{aligned}
$$

Here the last integral is independent of $|x|$ and the parameter $k$ in the hypothesis is chosen in such a way that the integral converges absolutely. Thus $\left|F^{-1}\left[e\left(v^{2}\right)\right](y)\right| \leqslant C|y|^{n(b-1)}$ and the assertion follows by the HardyLittlewood-Sobolev theorem (see [14], p. 119)

$$
\begin{aligned}
\left\|F^{-1}\left[e\left(v^{2}\right)\right] * f\right\|_{q} & \leqslant C N_{k+1}^{b}(e)\left\|\int_{R^{n}}|y|^{n(b-1)}|f(\cdot-y)| d y\right\|_{Q} \\
& \leqslant O^{\prime} N_{k+1}^{b}(e)\|f\|_{p} \quad(f \in S) .
\end{aligned}
$$

Our intention is to improve Theorem 1 by replacing $N_{k}^{b}(e)$ by a weaker integral condition and by diminishing the differentiation order. The latter can be achieved by introducing fractional derivatives: With J. Cossar 1941 define (cf. [17], p. 31) a fractional derivative of order $a, 0<a<1$, by

$$
e^{(\alpha)}(t)=-\lim _{u \rightarrow \infty} \frac{d}{d t} \int_{t}^{u}(s-t)^{-\alpha} e(s) d s,
$$

whenever the right-hand side exists, and fractional derivatives of higher order $a, a=k+\alpha$, by

$$
e^{(a)}(t)=\left(\frac{d}{d t}\right)^{k} e^{(a)}(t)
$$


Now introduce the classes $(b>0)$

$$
\begin{aligned}
\mathrm{WBV}_{1}^{b}=\left\{e \epsilon \mathrm{BV}_{\mathrm{loc}}(0, \infty) ; \lim _{t \rightarrow \infty} e(t)\right. & =e(\infty)=0 \\
|e|_{1, b} & \left.=\sup _{m} \int_{2^{m}}^{2^{m+1}}, t^{b}|d e(t)|<\infty, m \in Z\right\}
\end{aligned}
$$

and for $a>0$

$$
\begin{array}{r}
\mathrm{WBV}_{a+1}^{b}=\left\{e \epsilon C_{0}(0, \infty) ; e^{(a)}, \ldots, e^{(a-1)} \epsilon \mathrm{AC}_{\text {loo }}(0, \infty), e^{(a)} \epsilon \mathrm{BV}_{100}(0, \infty),\right. \\
\left.|e|_{a+1, b}=\sup _{m} \int_{2^{m}}^{2^{m+1}} t^{a+b}\left|d e^{(a)}(t)\right|<\infty, m \epsilon Z\right\} .
\end{array}
$$

(Note, $\mathrm{BV}_{a+1}^{b}$ in [2] and [18] is defined as above with $|e|_{a+1, b}$ replaced by $\int_{0}^{\infty} t^{a+b}\left|d e^{(a)}(t)\right| ; e \epsilon \mathrm{WBV}_{a+1}^{b}$ says that $e$ is weakly of bounded variation of order $a$ with respect to the weight $t^{b}$; see also the following lemma.)

LEMMVA 1. $\mathrm{WBV}_{a+1}^{b} \subset \mathrm{WBV}_{a^{\prime}+1}^{b}$ for $0 \leqslant a^{\prime}<a, b>0$.

Proof. $e \epsilon \mathrm{WBV}_{a+1}^{b}$ implies for each $e>0\left(m_{0}\right.$ denoting the largest integer such that $2^{m_{0}} \leqslant c$ )

$$
\begin{aligned}
\int_{c}^{\infty} t^{a}\left|d e^{(a)}(t)\right| & \leqslant \sum_{m_{0}}^{\infty} \int_{2^{m}}^{2^{m+1}} t^{a}\left|d e^{(a)}(t)\right| \\
& \leqslant|e|_{a+1, b} \sum_{m_{0}}^{\infty}\left(2^{m}\right)^{-b}<\infty
\end{aligned}
$$

Hence, by [17], Lemma 3.15, one has for each $t>0,-1 \leqslant a^{\prime}<a$, the representation

$$
e^{\left(a^{\prime}+1\right)}(t)=C_{a, a^{\prime}} \int_{t}^{\infty}(s-t)^{a-a^{\prime}-1} d e^{(a)}(s)
$$

and therefore,

$$
\begin{aligned}
& \int_{2^{m}}^{2^{m+1}} t^{a^{\prime}+b}\left|d e^{\left(a^{\prime}\right)}(t)\right| \leqslant C_{1} \int_{2^{m}}^{2^{m+1}}\left|d e^{(a)}(s)\right| \int_{2^{m}}^{s} t^{a^{\prime}+b}(s-t)^{a-a^{\prime}-1} d t+ \\
&+C_{2} \int_{2^{m}}^{2_{m}+1} t^{a^{\prime}+b} \sum_{i=m+1}^{\infty} \int_{2^{i}}^{2^{i+1}}(s-t)^{a-a^{\prime}-1}\left|d e^{(a)}(s)\right| d t \\
& \leqslant C_{3}|e|_{a+1, b}\left\{1+\int_{2^{m}} \sum_{i=m+1}^{\infty} 2^{-i} d t\right\} \leqslant C|e|_{a+1, b}
\end{aligned}
$$

uniformly in $m$, i.e. the assertion.

We are now able to deduce

THEOREM $1^{\prime}$. Let $0<b<1$, let $e \epsilon \mathrm{WBV}_{a+1}^{b n / 2}$ for some $a>n / 2-n b-$ $-1 / 2$ if $b \leqslant 1 / 2-1 / 2 n$ and $a \geqslant 0$ otherwise. Then $e\left(v^{2}\right) \in M_{p}^{q}$, where $1<p$ $<q<\infty$ and $1 / q=1 / p-b$.
Proof. The above lemma gives us the representation (2.6) for $e=e^{(0)}$ and hence

$$
F^{-1}\left[e\left(v^{2}\right)\right](x)=O \int_{0}^{\infty} s^{a} s^{n / 2} r_{a}(\sqrt{s} x) d e^{(a)}(s),
$$

because

$$
\left|F^{-1}\left[e\left(v^{2}\right)\right](x)\right|
$$

$$
\leqslant C|x|^{n(b-1)}|e|_{a+1, b n / 2} \sum_{-\infty}^{\infty} \sup _{2^{m} \leqslant s \leqslant 2^{m+1}}(\sqrt{s}|x|)^{n(1-b)}\left|r_{a}(\sqrt{s}|x|)\right|
$$

with uniformly convergent sum (when the parameter $a$ is chosen as in the hypothesis), since on account of (2.1) the single terms of the sum are dominated by $C\left(\sqrt{2^{m}}|x|\right)^{n(1-b)} \min \left\{1,\left(\sqrt{2^{m}}|x|\right)^{-a-(n+1) / 2}\right\}$. The assertion now follows as in Theorem 1.

What is annoying about Theorem $1^{\prime}$ is the fact that given a radial function $e(|v|)$ one has to examine $e(\sqrt{t})$. In the case $a=k$ integer, it easily follows with the aid of Lemma 1 that $|e(\sqrt{t})|_{a+1, b n / 2} \leqslant C M$ if $|e(t)|_{a+1, b n} \leqslant M$. Hence

CoROLLARY. Let $0<b<1, e(t)$ be a function on $(0, \infty)$ with

$$
e(\infty)=0, \quad \sup _{m} \int_{2^{m}}^{2^{m+1}} t^{k+b n}\left|d e^{(k)}(t)\right|<\infty
$$

for $k$ as in Theorem 1. Then $e(|v|) \in M_{p}^{q}, 0<1 / q=1 / p-b, p>1$.

Remark. a) Let us mention that the assumption $b>0$ allows this straightforward reduction of the multiplier problem to the Hardy-Littlewood-Sobolev theorem. The latter theorem is an easy consequence (see Hedberg [5]) of the fact that the spherical maximal function is a bounded operator on $L^{p}, p>1$ (see e.g. [14], p. 5). ।

b) Hörmander [6] proved: If $1<p \leqslant 2 \leqslant q<\infty$, then $|v|^{b n} e(v) \epsilon L^{\infty}$ implies $e(v) \epsilon M_{p}^{q}(e(v)$ not necessarily radial). It is clear that this criterion in the prescribed $(p, q)$-range is much better than Theorem $1^{\prime}$. Interpolation between these two results should give an improvement of Theorem $1^{\prime}$ with respect to differentiation order.

c) The straightforward estimate $\left|F^{-1}\left[e\left(v^{2}\right)\right](y)\right| \leqslant C|y|^{n(b-1)}$ has the further advantage that one can directly apply weighted versions of the Hardy-Littlewood-Sobolev theorem due to Stein-Weiss [15] and Muckenhoupt-Wheeden [12].

THEOREM $1^{\prime \prime}$. Let $0<b<1$ and $e(t)$ satisfy the conditions of Theorem $1^{\prime}$.

(i) [15] If $1<p \leqslant q<\infty, \alpha<n / p^{\prime}, \beta<n / q, \alpha+\beta \geqslant 0$, and $1 / q$ $=1 / p-b+(\alpha+\beta) / n$, then

$$
\left\|F^{-1}\left[e\left(v^{2}\right)\right] * f(x)|x|^{-\beta}\right\|_{q} \leqslant C\left\|f(x)|x|^{\alpha}\right\|_{p} .
$$


(ii) [12] Assume $1<p<q<\infty, 1 / q=1 / p-b$, and $V(x)$ is a nonnegative function on $R^{n}$ such that for every cube, $Q(|Q|$ denotes the Lebesgue measure of $Q$ ),

$$
\left(\frac{1}{|Q|} \int_{Q}[V(x)]^{q} d x\right)^{1 / q}\left(\frac{1}{|Q|} \int_{Q}[V(x)]^{-p^{\prime}} d x\right)^{1 / p^{\prime}} \leqslant K \quad\left(\frac{1}{p}+\frac{1}{p^{\prime}}=1\right),
$$

$K$ independent of $Q$. Then there is a $C$, independent of $f$, such that

$$
\left\|F^{-1}\left[e\left(v^{2}\right)\right] * f(x) V(x)\right\|_{q} \leqslant C\|f(x) V(x)\|_{p} .
$$

d) It is interesting to note what happens in the limit cases $p=1$ and $b=0$.

If $p=1$, then $b=1 / q^{\prime}$, and Theorem 1 in [2] tells us:

Let $e \epsilon \mathrm{BV}_{a+1}^{b n / 2}$, i.e. essentially

$$
e(\infty)=0, \quad \int_{0}^{\infty} t^{a+b n / 2}\left|d e^{(a)}(t)\right|<\infty,
$$

with $a$ as in Theorem $1^{\prime}$. Then $e\left(v^{2}\right) \in M_{1}^{q}, 0 \leqslant 1 / q=1-b$.

This theorem nicely shows how much stronger we have to choose our hypotheses in order to cover also the case $p=1$. The case $b=0$ is treated in the following section.

3. Radial multipliers $(p=q)$. Here it is convenient to introduce the classes

$$
\mathrm{WBV}_{1}=\left\{e \epsilon L^{\infty}(0, \infty) ;|e|_{1}=\sup _{m \in Z} \int_{2^{m}}^{2^{m+1}}|d e(t)|<\infty\right\}
$$

and for $a>0$

$$
\begin{gathered}
\mathrm{WBV}_{a+1}=\left\{e \epsilon L^{\infty}(0, \infty) ; e^{(a)}, \ldots, e^{(a-1)} \in \mathrm{AO}_{\mathrm{loc}}(0, \infty), e^{(a)} \in \mathrm{BV}_{\mathrm{loc}}(0, \infty),\right. \\
\left.|e|_{a+1}=\sup _{m \in Z} \int_{2^{m}} t^{a}\left|d e^{(a)}(t)\right|<\infty\right\} .
\end{gathered}
$$

(Note, $\mathrm{BV}_{a+1}$ in [17] is defined as above with $|e|_{a+1}$ replaced by $\left.\int_{0}^{\infty} t^{a}\left|d e^{(a)}(t)\right|.\right)$ We want to show a result analogous to Lemma 1 . But this is now more difficult since in general (2.5) does not hold under our new hypothesis.

LEMMA 2. $\mathrm{WBV}_{a+1} \subset \mathrm{WBV}_{a^{\prime}+1}$ for $0 \leqslant a^{\prime}<a$.

Proof. If $a^{\prime}$ and $a$ are both integers, then $e \epsilon \mathrm{WBV}_{a+1}$ clearly implies for each $c>0, t>0, k=1, \ldots, a(\operatorname{see}(2.6))$

$$
\int_{c}^{\infty} t^{a-1}\left|d e^{(a)}(t)\right|<\infty, \quad e^{(k)}(t)=C \int_{t}^{\infty}(s-t)^{a-k} d e^{(a)}(t)
$$

and the assertion follows as in the proof of Lemma 1 . In the case of fractional $a^{\prime}, a$, the same idea is used. Since the proof is quite parallel to the corresponding one in [17], Section 3.3.1, we only sketch it. Define for $a>0$

$$
I_{u}^{a} f(t)=C \int_{i}^{u}(s-t)^{a-1} f(s) d s \quad(0<t<u)
$$

and $I^{a} f(t)=\lim _{u \rightarrow \infty} I_{u}^{a} f(t)$ if the limit exists.

First consider $e \epsilon \mathrm{WBV}_{a+1}$ for $0<a \leqslant 1$; then one can show $I_{u}^{a}\left(e^{(a)}\right)(t)$ $=O(1)$ uniformly in $u$, which is used to deduce

$$
e^{(a)}(t)=-\int_{t}^{\lceil\infty} d e^{(a)}(s)
$$

As in [17], pp. 34-35, it follows for each $a^{\prime}, 0 \leqslant a^{\prime}<a$, that $e^{\left(a^{\prime}\right)}(t)$ $e^{\left(a^{\circ}+1\right)}(t)$ exist almost everywhere and

$$
e^{(r)}(t)=C \int_{i}^{\infty}(s-t)^{a-r} d e^{(a)}(s) \quad\left(0<r \leqslant a^{\prime}+1\right)
$$

for almost all $t$. Since $\left(e^{\left(a^{\prime}\right)}\right)^{\left(a-a^{\prime}\right)}(t)=e^{(a)}(t)$ a.e., the assertion now follows in case $0 \leqslant a^{\prime}<a \leqslant 1$ analogously to Lemma 1 . If $a>1$, one first shows (3.1) (see [17], p. 36), hence $\mathrm{WBV}_{a+1} \subset \mathrm{WBV}_{a}$ and iteratively $\mathrm{WBV}_{a-k+1}$ $\subset \mathrm{WBV}_{a-k}$ with $0<a-k \leqslant 1$. Applying the known results in the latter case leads to (2.6) for $-1<a^{\prime}<a$ (see [17], p. 37), and hence the assertion for arbitrary $0 \leqslant a^{\prime}<a$.

THEOREM 2. Let e $\epsilon \mathrm{WBV}_{a+1}$ for some $a>(n-1) / 2$. Then $e\left(v^{2}\right) \epsilon M_{p}^{p}$, $1<p<\infty$.

The proof differs from that of Theorem $1^{\prime}$ for we do not have (2.6) for $a^{\prime}=-1$ at our disposal. But all we need is (2.7). To this end, let $G(t) \epsilon C^{\infty}$ with $G(t) \equiv 1$ for $0 \leqslant t \leqslant 1, G(t) \equiv 0$ for $2 \leqslant t<\infty$, and $0 \leqslant G(t)$ $\leqslant 1$ otherwise, and consider

$$
e_{u}(t)=e(t) G\left(\frac{t}{u}\right) \quad(u>0) .
$$

Then $e_{u} \epsilon \mathrm{WBV}_{a+1}$ uniformly in $u>0$. For integral $a$ this follows immediately by Leibniz' rule using

$$
\left|\left(\frac{t}{u}\right)^{a^{\prime}} G^{\left(a^{\prime}\right)}\left(\frac{u}{t}\right)\right| \leqslant 0 \int_{0}^{\infty} t^{a^{\prime}}\left|G^{\left(a^{\prime}+1\right)}(t)\right| d t<\infty
$$

for arbitrary $a^{\prime} \geqslant 0$ (see e.g. [18]) and Lemma 2. For fractional $a^{\prime}$ proceed analogously to [18], Lemma 1.1(iv), where one has again to use (3.2) and Lemma 2. 
Now $e_{u}(t)=0$ for $t \geqslant 2 u$; therefore, (2.6) holds for each $e_{u}\left(a^{\prime}=-1\right)$ and hence (2.7) for $x \neq 0$ with $e$ replaced by $e_{u}$ (take in the proof of Theorem 1 only $f \epsilon S$ with support disjoint from the origin), because

$$
\left|F^{-1}\left[e_{u}\left(v^{2}\right)\right](x)\right| \leqslant C|x|^{-n}\left|e_{u}\right|_{a+1} \sum_{-\infty}^{\infty} \sup _{2^{m} \leqslant s \leqslant 2^{m+1}}(\sqrt{s}|x|)^{n}\left|r_{a}(\sqrt{s}|\infty|)\right|
$$

with the sum uniformly convergent in $x \neq 0$ by $(2.1)$ if $a>(n-1) / 2$.

Now $e_{u}\left(v^{2}\right) \rightarrow e\left(v^{2}\right)$ bounded and pointwise, i.e. in $\boldsymbol{S}^{\prime}$, and therefore $F^{-1}\left[e_{u}\left(v^{2}\right)\right]$ tends in $S^{\prime}$ towards $F^{-1}\left[e\left(v^{2}\right)\right]$. On the other hand, for $f \in S$ with support disjoint from the origin,

$$
\left\langle F^{-1}\left[e_{u}\left(v^{2}\right)\right], f\right\rangle \rightarrow \int_{R^{n}} C \int_{0}^{\infty} s^{a} s^{n / 2} r_{a}(\sqrt{s}|x|) d e^{(a)}(s) f(x) d x,
$$

i.e. for $x \neq 0$ the distribution $F^{-1}\left[e\left(v^{2}\right)\right]$ has the representation (2.7). It is not hard to check that Hörmander's condition

$$
t^{-n} \int_{|x| \geqslant 2}\left|F^{-1}\left[e\left(v^{2}\right)\right]\left(\frac{x-y}{t}\right)-F^{-1}\left[e\left(v^{2}\right)\right]\left(\frac{x}{t}\right)\right| d x \leqslant C
$$

is satisfied for $|y| \leqslant 1$, all $t>0$ and $a>(n-1) / 2$. Since $e\left(v^{2}\right) \in L^{\infty}\left(R^{n}\right)$, i.e. $e\left(v^{2}\right) \in M_{2}^{2}$, the assertion follows by Hörmander [6], Theorem 2.1.

By the above, it is clear that for $a>(n-1) / 2$

$$
\left|F^{-1}\left[e\left(v^{2}\right)\right](x)\right| \leqslant \frac{C}{|x|^{n}}|e|_{a+1} .
$$

Furthermore, by assumption,

$$
\left|e\left(v^{2}\right)\right| \leqslant M .
$$

In order to apply a recent result of Coifman-Fefferman [4] on singular integrals in weighted $L^{p}$-spaces it remains to prove that for $|y|<|x| / 2$

$$
\left|F^{-1}\left[e\left(v^{2}\right)\right](x)-F^{-1}\left[e\left(v^{2}\right)\right](x-y)\right| \leqslant C \frac{|y|}{|x|^{n+1}} .
$$

By Taylor's formula and the representation (2.7) this can be shown to be true for $a>(n+1) / 2$, i.e., the differentiation order is increased by one.

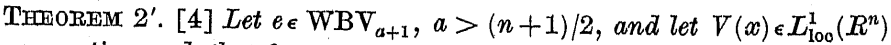
be non-negative such that for every cube, $Q$,

$$
\left(\frac{1}{|Q|} \int_{Q} V(x) d x\right)\left(\frac{1}{|Q|} \int_{Q}^{0} V(x)^{-1 /(p-1)} d x\right)^{p-1} \leqslant K,
$$

$K$ being independent of $Q$. Then, for $1<p<\infty$,

$$
\int_{R^{n}}\left|F^{-1}\left[e\left(v^{2}\right)\right] * f(x)\right|^{p} \nabla(x) d x \leqslant C_{p} \int_{R^{n}}|f(x)|^{p} \nabla(x) d x .
$$

Again the question arises whether Theorem 2 and $2^{\prime}$ remain true for $e(|v|)$. We only treat the case $a$ being an integer.

LeMma $2^{\prime}$. Let $\Phi(t)$ be a non-negative, strictly monotone increasing function with $\lim _{t \rightarrow 0} \Phi(t)=0$ and $\lim \Phi(t)=\infty$, and let $\Phi$ possess $(a+1)$ continuous derin on $(0, \infty)$ with

$$
\left|t^{i} \Phi^{(i+1)}(t)\right| \leqslant D \Phi^{\prime}(t) \quad(i=0, \ldots, a) .
$$

Then $e(t) \epsilon \mathrm{WBV}_{a+1}$ implies $e(\Phi(t)) \epsilon \mathrm{WBV}_{a+1}$.

Proof. Analogous to [17], p. 28, it easily follows that

$$
\int_{2^{m}}^{2^{m+1}} t^{a}\left|d\left(\frac{d}{d t}\right)^{a} e(\Phi(t))\right| \leqslant C \int_{\Phi\left(2^{m}\right)}^{\Phi\left(2^{m+1}\right)} t^{a}\left|d e^{(a)}(t)\right| .
$$

Now $\Phi$ satisfies the so-called $\Delta_{2}$-condition: $\Phi(2 t) \leqslant K \Phi(t)$ for all $t>0$ (see [7], p. 24), because the hypothesis implies

$$
t \Phi^{\prime}(t) \leqslant(D+1) \Phi(t)
$$

(see [17], p. 29), and hence

$$
\log \frac{\Phi(2 t)}{\Phi(t)}=\int_{i}^{2 t} \frac{\Phi^{\prime}(s)}{\Phi(s)} d s \leqslant \int_{i}^{2 t} \frac{D+1}{s} d s=\log 2^{D+1} .
$$

This $\Delta_{2}$-condition allows the final estimate

$$
\int_{\Phi\left(2^{m}\right)}^{\Phi\left(2^{m+1}\right)} t^{a}\left|d e^{(a)}(t)\right| \leqslant \int_{K^{i} \Phi(1)}^{K^{i+2} \Phi(1)} t^{a}\left|d e^{(a)}(t)\right| \leqslant C^{\prime}|e|_{a+1}
$$

Corolrary. Let $\Phi$ be as in Lemma $2^{\prime}$ and

$$
|e(t)| \leqslant M, \quad \sup _{m} \int_{2^{m}}^{2^{m+1}} t^{k}\left|d e^{(k)}(t)\right|<\infty
$$

for integral $k>(n-1) / 2$. Then $e\left(\Phi\left(v^{2}\right)\right) \in M_{p}^{p}, 1<p<\infty$. In particular, $e(|v|) \in M_{p}^{p}$.

The result $e(|v|) \in M_{p}^{p}$ is essentially due to Bonami-Clerc [1]; for compact Lie groups see Clerc [3]. Let us observe that a sufficient condition for radial $M_{1}^{1}$-multipliers is

$$
|e(t)| \leqslant M, \quad \int_{0}^{\infty} t^{a}\left|d e^{(a)}(t)\right|<\infty \quad(a>(n-1) / 2)
$$

(see [17], Theorem 5.10). 
4. Arbitrary multipliers $(1<p<q<\infty)$. If $e(v)$ is not necessarily radial, there are $M_{p}^{q}$ multiplier criteria $(p<q)$ due to Lizorkin $[10]$, [11] and Littman [9] (the latter one is based upon Hörmander's $M_{p}^{p}$ multiplier criterion). Here we want to prove a special case of Lizorkin's results by a direct reduction to the coordinatewise fractional integral $(0<b<1)$

$$
R^{b} f(x)=\int_{R^{n}}\left|y_{1}\right|^{b-1} \cdots\left|y_{n}\right|^{b-1} f(x-y) d y,
$$

which satisfies for some constant $O$ independent of $f \epsilon S$

$$
\left\|R^{b} f\right\|_{q} \leqslant C\|f\|_{p} \quad(1<p<q<\infty, 1 / q=1 / p-b) .
$$

(see [13], Theorem 5.2.12)

THEOREM 3. Let $0<b<1, e(v)$ be one time differentiable on $v_{i} \neq 0$, $1 \leqslant i \leqslant n$, such that the differentiation order may be interchanged and

$$
\prod_{i=1}^{n}\left|v_{i}\right|^{j_{i}+b}\left|D^{j} e(v)\right| \leqslant M
$$

is uniformly bounded for $v_{i} \neq 0$, where the coordinates $j_{i}$ of the multiindex $j$ are 0 or 1 . Then $e(v) \in M_{p}^{q}$ with $1<p<q<\infty, 1 / q=1 / p-b$.

Proof. (i) One may restrict oneself to functions $e(v)$ even in each coordinate: For, as may be seen by induction, one can decompose $e(v)$ into $2^{n}$ terms $e_{i}(v)$ with $e_{i}(v)$ even in $n^{\prime}$ coordinates, $0 \leqslant n^{\prime} \leqslant n$, and odd in the remaining $n^{\prime \prime}$ ones, $n^{\prime}+n^{\prime \prime}=n$. Further,

$$
e_{i}(v)=2^{-n} \sum \pm e\left( \pm v_{1}, \ldots, \pm v_{n}\right),
$$

where the sum is extended over all \pm combinations of the $n$ variables $v_{1}, \ldots, v_{n}$, hence consisting of $2^{n}$ terms. Now, choosing $\operatorname{sgn} v_{i}$ appropriately: $e_{i}(v)=\operatorname{sgn} v_{i_{1}} \ldots \operatorname{sgn} v_{i_{n^{\prime \prime}}} e_{i}^{*}(v)$, one obtains functions $e_{i}^{*}$ even in each coordinate.

Defining for $f \epsilon S, C$ a suitable constant,

$$
f_{i}(x)=O \mathrm{PV} \int_{-\infty}^{\infty} \ldots \int_{-\infty}^{\infty} f\left(x-t_{1} e^{i_{1}}-\ldots-t_{n^{\prime \prime}} e^{i_{n^{\prime \prime}}}\right) t_{1}^{-1} \ldots t_{n^{\prime \prime}}^{-1} d t_{1} \ldots d t_{n^{\prime \prime}}
$$

$\left(e^{i_{1}} \epsilon R^{n}\right.$ being the unit vector on the $i_{1}$-axis), one lnnows that $f_{i}(x)$ existis in $L^{p}, 1<p<\infty$, and has Fourier transform

Hence, for $f \in S$,

$$
\operatorname{sgn} v_{i_{1}} \ldots \operatorname{sgn} v_{i_{n^{\prime \prime}}} f^{\wedge}(v) \text {. }
$$

$$
F^{-1}\left[e f^{\wedge}\right]=\sum_{i=1}^{2^{n}} F^{-1}\left[e_{i} f^{\wedge}\right]=C \sum_{i=1}^{2^{n}} F^{-1}\left[e_{i}^{*}\right] * f_{i},
$$

and we have to discuss $e_{i}^{*}$. (ii) Set $e_{i}^{*}(v)=h(v)$. By (4.2) it follows that

(4.4) $\left|v_{1}\right|^{j_{1}+b} \ldots\left|v_{n}\right|^{j_{n}+b}\left|D^{j} h(v)\right| \leqslant\left|v_{1}\right|^{j_{1}+b} \ldots\left|v_{n}\right|^{j_{n}+b}\left|D^{j} e(v)\right| \leqslant M$,

i.e., $h(v)\left(=e_{i}^{*}(v)\right.$ for arbitrary $\left.i, 1 \leqslant i \leqslant 2^{n}\right)$ satisfies the hypotheses of the theorem. In particular, since $h(v)$ is even in each coordinate, it follows by partial integration that

$$
h(v)=C \int_{\left|v_{1}\right|}^{\infty} \ldots \int_{\left|v_{n}\right|}^{\infty} D^{j} h(u) d u \quad\left(j=(1, \ldots, 1), v_{i} \neq 0\right) .
$$

Evaluating the inverse Fourier transform (analogous to Theorem 1 ) leads to $(j=(1, \ldots, 1))$

$$
\begin{aligned}
& F^{-1}[h](x)=C \int_{0}^{\infty} \ldots \int_{0}^{\infty} \prod_{i=1}^{n} \frac{\sin x_{i} u_{i}}{x_{i}} D^{j} h(u) d u \\
= & C\left|x_{1}\right|^{b-1} \ldots\left|x_{n}\right|^{b-1} \int_{0}^{\infty} \ldots \int_{0}^{\infty} u_{1}^{1+b} \ldots u_{n}^{1+b} D^{j} h(u) \cdot \prod_{i=1}^{n} \frac{\sin \left|x_{i}\right| u_{i}}{\left(\left|x_{i}\right| u_{i}\right)^{1+b}}\left|x_{i}\right| d u_{i} .
\end{aligned}
$$

Hence, by (4.4),

$$
\left|F^{-1}\left[e_{i}(v)\right](x)\right| \leqslant C^{\prime} M\left|x_{1}\right|^{b-1} \ldots\left|x_{n}\right|^{b-1}
$$

uniformly in $i, 1 \leqslant i \leqslant 2^{n}$, and therefore, by (4.1) and (4.3),

$$
\left\|F^{-1}\left[e f^{\wedge}\right]\right\|_{q} \leqslant C^{\prime} M \sum_{i=1}^{2^{n}}\left\|f_{i}\right\|_{p} \leqslant C M\|f\|_{p}
$$

$C$ being independent of $f \epsilon S, b, p ; q$ being as in the hypotheses.

Analogous to Theorem $1^{\prime}$ one may weaken the hypotheses: introduce a dyadic decomposition of $R^{n}$ as is done on p. 103 in [14], i.e., decompose $R^{n}$ into disjoint rectangles $\Delta$ which are Cartesian products of intervals $\left[2^{i}, 2^{i+1}\right]$ and $\left[-2^{i+1},-2^{i}\right]$. Now regard for every $n^{\prime} \leqslant n, R^{n^{\prime}}$ be embedded in $R^{n}$ in the obvious way: $R^{n^{\prime}}$ is the subspace of all points of the form $\left(x_{1}, \ldots, x_{n^{\prime}}, 0, \ldots, 0\right)$.

THMORIM 3 . Let $0<b<1$, e(v) be continuous for $v_{k} \neq 0$, one time differentiable in each coordinate such that the differentiation order may be interchanged. ITurther,

$$
\begin{aligned}
& \text { (i) }\left|v_{1}\right|^{b} \ldots\left|v_{n}\right|^{b}|e(v)| \leqslant M, \\
& \text { (ii) } \sup _{v_{n^{\prime}+1}, \ldots, v_{n}} \int_{\Delta}\left|v_{1}\right|^{b} \ldots\left|v_{n^{\prime}}\right|^{b}\left|\frac{\partial^{n^{\prime}} e(y)}{\partial v_{1} \ldots \partial v_{n^{\prime}}}\right| d v_{1} \ldots d v_{n^{\prime}} \leqslant M
\end{aligned}
$$

$\left(0<n^{\prime} \leqslant n\right.$, if $n^{\prime}=n$ the "sup" sign is omitted $)$ as $\triangle$ ranges over dyadic rectangles of $R^{n !}$.

(iii) The condition analogous to (ii) is valid for every one of the $n$ ! permutations of the variables $v_{1}, \ldots, v_{n}$. 
Then $e(v) \in M_{p}^{q}$, where $1<p<q<\infty$ and $1 / q=1 / p-b$.

The proof is only a combination of the methods applied in the proofs of Theorems $1^{\prime}$ and 3 ; therefore we omit it.

Remark. a) As already mentioned, Theorem 3 is due to Lizorkin [10], [11] who obtains it as a special case of sharper results. Since his methods cover also the case $b=0$, his argumentation is naturally more complicated. Lizorkin's basic idea consists in the supersition of some 'elementary' transformations, essentially coordinatewise Riesz potentials and Hilbert transforms. Our method is a modification of Lizorkin's one for we emphasize the important role played by partial integrations (by which the Fourier transforms of Bochner-Riesz kernels are generated) and give a direct reduction of the multiplier problem to problems concerning weakly singular and singular integrals.

b) A remark analogous to the first Remark a) holds (one has to replace the spherical maximal function by the maximal function with respect to rectangles whose sides are parallel to the axis; see [14], p. 24).

c) There exist variants of the Hardy-Littlewood-Sobolev theorem with coordinatewise weights (see e.g. Okikiolu [13], Theorem 4.5.14). These allow us to formulate an analogue to Theorem $1^{\prime \prime}(\mathrm{i})$, but we omit it.

d) There remains the question what happens in the limit cases $p=1$ and $b=0$.

If $p=1$, then $b=1 / q^{\prime}$ and a result in [18] tells us: Under the hypotheses of Theorem 3 , where

$\int_{\Delta}|\ldots| d v_{1} \ldots d v_{n^{\prime}} \quad$ is to be replaced by $\int_{-\infty}^{\infty} \ldots \int_{-\infty}^{\infty}|\ldots| d v_{1} \ldots d v_{n^{\prime}}$, it follows that $e(v) \in M_{1}^{q}$, where $1<q \leqslant \infty$ and $1 / q=1-b$.

Theorem $3^{\prime}$ remains valid when setting formally $b=0$ (see [14], Theorem $6^{\prime}, \mathrm{IV}$ ). This is the standard Marcinkiewicz Fourier multiplier criterion on $L^{p}\left(R^{n}\right), 1<p<\infty$, due to Krée [8].

\section{References}

[1] A. Bonami and J. L. Clerc, Sommes de Cesàro et multiplicateurs des dévelop. pements en harmonique sphériques, Trans. Amer. Math. Soc. 183 (1973), pp. 223-264.

[2] P. L. Butzer, R. J. Nessel and W. Trebels, On radial M Mourier multipliers, in: Math. Struct., Comput. Math., Math. Modelling, pp. 187-193, Sofia 1975.

[3] J.-L. Clerc, Sommes de Riesz et multiplicateurs sur un groupe de Lie compact, Ann. Inst. Fourier (Grenoble) 24, 1 (1974), pp. 149-172.

[4] R. R. Coifman and C. Fefferman, Weighted norm inequalities for maximal functions and singular integrals, Studia Math. 51 (1974), pp. 241-250.

[5] L. I. Hedberg, On certain convolution inequalities, Proc. Amer. Math. Soc. 36 (1972), pp. 505-510.
[6] L. Hörmander, Estimates for translation invariant operators in $L^{p}$ spaces, Acta Math. 104 (1960), pp. 93-140.

[7] M. A. Krasnosel'skil and Ya. B. Rutickiľ, Convex functions and Orlice spaces, Gröningen 1961

[8] P. Krée, Sur les multiplicateurs dans $F L^{p}$, Ann. Inst. Fourier (Grenoble) 16 (1966), pp. 31-89.

[9] W. Littman, Multipliers in $L^{p}$ and interpolation, Bull. Amer. Math. Soc. 71 (1965), pp. 764-766.

[10] P. I. Lizorkin, $\left(L_{p}, L_{q}\right)$-multipliers for Fourier integrals, Soviet Math. Dokl. 4 (1963), pp. 1420-1424.

[11] - On multipliers of Fourier integrals in the spaces $L_{p, \theta}$, Proc. Steklov Inst. Math. 89 (1967), pp. 269-290.

[12] B. Muckenhoupt and R. Wheeden, Weighted norm inequalities for fractional integrals, Trans. Amer. Math. Soc. 190 (1974), pp. 1-15.

[13] G. O. Okikiolu, Aspects of the theory of bounded integral operators in $L^{p_{-s p a c e s}}$ Academic Press, New York 1971.

[14] E. M. Stein, Singular integrals and differentiability properties of functions, Princeton 1971 .

[15] - and G. Weiss, Fractional integrals on n-dimensional Euclidean space, J. Math. Mech. 7 (1958), pp. 503-514.

[16] - - Introduction to Fourier analysis on Euclidean spaces, Princeton 1971.

[17] W. Trebels, Multipliers for $(C, a)$-bounded Fourier expansions in Banach spaces and approximation theory, Springer Leçture Notes in Math. 329, Berlin 1973.

[18] - Some Fourier multiplier oriteria and the spherical Bochner-Riesz kernel, Rev. Roumaine Math. Pure Appl. 20 (1975), pp. 1173-1185.

\section{F. B. MATHEMATIK}

F. MATHATIK 